\title{
From the journals
}

\section{Warnings on use of selective serotonin reuptake inhibitors (SSRIs) and serotonin- norepinephrine reuptake inhibitors (SNRIs) in children and adolescents}

The European Medicines Agency, following a review of SSRIs has concluded that suicide related behaviour and hostility were observed more frequently in children and adolescents receiving these antidepressants than those receiving a placebo. Doctors are advised that these antidepressants should not be used for treatment of children and adolescents except for their approved indications, which, for some of these antidepressants, include obsessive-compulsive disorder and attention deficit hyperactivity disorder. Reactions 2005; 1049: 2

\section{Non-steroidal anti-inflammatory drug (NSAID) use is associated with acute renal failure}

Study of 386916 patients aged 50-84 years in the General Practice Research Database of the United Kingdom identified 103 patients with idiopathic acute renal failure. They were compared with 5000 controls matched for age, sex and calendar year. The incidence rate was 1.1 cases of non-fatal acute renal failure per 10000 person years in the study.

The risk of developing first ever acute renal failure was greater for current NSAID users than for nonusers and the risk decreased following NSAID discontinuation. The relative risk was higher for current NSAID users with a history of hypertension, heart failure or who used concomitant diuretics or calcium channel antagonists. Reactions 2005; 1049: 4

\section{Post-traumatic stress}

After surviving a natural disaster such as the recent tsunami, people need basics, e.g. food, shelter, medical care and consolation. Some add psychological care to this list of basic needs. Doctors should know whether and when psychological help is necessary. Following trauma, it is common to have symptoms such as flashbacks, sleep problems, difficulty in concentration and emotional instability. Usually these symptoms are mild and may last for about 4 weeks. The National Institute for Clinical Excellence (NICE) recommends initial watchful waiting without any psychological intervention during this period. According to the NICE guidelines, treatment is necessary when, following a disaster, a person develops post-traumatic stress disorder, depression, suicidality, addiction, medically unexplained physical symptoms or dissociative disorders.

In post-traumatic stress disorder, there is deregulation of the fear system. Fear is a necessary emotion at times of danger, to initiate survival behaviour. Once the trauma is over, the fear system normally calms down after a few days or weeks. In post-traumatic stress disorder this system fails to reset to normal, keeping the sufferer hyperalert. The disorder is characterised by involuntary, persistent remembering or reliving the traumatic event in flashbacks.

For post-traumatic stress disorder NICE recommends trauma-focused psychological therapy. Using drugs as firstline treatment is not recommended. The most effective treatment for resetting the fear system is cognitive behaviour therapy. By imaginary exposure to the traumatic event the fear reaction will decrease in time. British Medical Journal 2005; 330: 1038-9

\section{Caring for people with chronic kidney disease}

Many studies have shown that the presence of chronic kidney disease is an independent and significant cardiovascular risk factor in the general population as well as in those with pre-existing cardiovascular disease. Patients with potentially reversible causes of kidney failure or those whose kidney function is deteriorating rapidly need to be identified and referred for rapid assessment by a nephrologist. The use of calculated GFR is likely to identify patients with less severe degrees of chronic kidney disease. These patients may be missed if serum creatinine alone is used to assess kidney function.

Patients with a GFR below $15 \mathrm{~mL} / \mathrm{min} / 1.73 \mathrm{~m}^{2}$ need to be referred to a nephrologist. Those with a GFR of $15-30 \mathrm{~mL} / \mathrm{min} / 1.73 \mathrm{~m}^{2}$ may be managed by a specialist clinic with support from a nephrologist. At this stage, anaemia and hyperparathyroidism need management using practice guidelines. The large number of patients with a GFR of $30-60 \mathrm{~mL} / \mathrm{min} / 1.73 \mathrm{~m}^{2}$ may be managed by the general practitioner with a specialist interest as long as they are able to work to guidelines, and have ready access to advice from a nephrologist. These patients need basic interventions. The aims should be to prevent progression of chronic kidney disease and prevent cardiovascular disease. The interventions include tight control of blood pressure, correcting lipid abnormalities and lifestyle changes including smoking. Targets for control should be set for blood pressure, diabetes, lipids and smoking. British Medical Journal 2005; 330: $1039-40$. 


\section{Transient ischaemic attacks (TIA) are not just a warning of stroke}

In a prospective, multicentre, observational study done in southwest Germany, 1380 TIA patients were compared with 3855 stroke patients. The study revealed that during the hospital stay stroke incidence was $8 \%$ for TIA patients and another 5\% within the first 6 months. For patients with ischaemic strokes(IS) these figures were $7 \%$ and $6 \%$ respectively. Two percent of TIA patients died in hospital (5\% afterward) compared with $9 \%$ of stroke patients (10\% afterward). Seventeen patients with TIA compared with $38 \%$ patients with IS were dependent at follow up. Admission to a stroke unit was found to be a valid predictor for survival and independence.

There is a relevant individual risk of early stroke, death or disability in TIA patients. Transient ischaemic attacks should be investigated in the same way as strokes. The management and treatment strategies should be similar in both TIA and acute stroke. Stroke 2004; 35: 2453-8.

Kusum de Abrew, Senior Lecturer, Department of Pharmacology, Faculty of Medicine, Colombo. (Competing interests: none declared).

\section{Direct to consumer advertising of medicinal drugs}

The public is ill served when governments allow promotion of prescription drugs that stretches the limits of the law - and beyond. No country has been successful at regulating any type of direct to consumer advertising to ensure the public obtains reliable balanced information on drug benefits and risks. Repeated breaches by companies speak for themselves. The potential awareness raising benefits of direct to consumer advertising could be better targeted and sustained at lower cost with less harm through publicly funded and accountable drug information services and health campaigns.

PR Mansfield, B Mintzes, D Richards, L Toop. British Medical Journal 2005; 330: 5-6 (Editorial) 